\title{
EL PLAN DE MEJORA DEL HÁBITAT MINERO DE ASTURIAS (1966-1969) BASES, CONTEXTO Y BALANCE TERRITORIAL
}

\author{
The plan of improvement of the mining habitat of Asturias \\ (1966-1969) bases, context and territorial balance
}

\author{
Faustino Suárez Antuña
}

Recibido 09/10/2007; aceptado el 15/01/2008

Resumen. El fin del periodo autárquico dejaba las comarcas mineras asturianas en una difícil situación. Las estructuras productivas estaban obsoletas, lo que dificultaba la competitividad de su carbón y acero en un nuevo contexto de mercado libre y abierto. Pero más acuciante aún era lo que se refería a su tejido urbano y social. En los años sesenta del siglo XX muchos lugares carecían aún de infraestructuras básicas como carreteras, luz o agua, y todas las cuencas presentaban escasos equipamientos educativos, deportivos, $y$ culturales. El Plan de Mejora del Hábitat Minero intentó corregir estos desequilibrios antes de abordar la reestructuración de sus sectores industriales principales.

Palabras clave: Hábitat minero, Autarquía, Asturias, urbanismo, desarrollo regional, infravivienda.

Abstract. The autarkic period end left the Asturian mining regions in a difficult situation. Production structures were obsolete, leaving little room to the competitiveness of its coal and steel in a new context of free and open trade. But even more pressing it was regarding its urban and social fabric. In the Sixties of the twentieth century many places still lacked basic infrastructures like roads, electricity or water, and all the basins had little education facilities, sports, and libraries. The Mining Habitat Improvement Plan of will try to correct these imbalances before addressing the restructuring of its main industrial sectors.

Key Words: mining habitat, autarky, Asturias, town planning, regional development, infrahouse.

\section{INTRODUCCIÓN}

Las comarcas mineras, y especialmente las asturianas por la mayor densidad de trabajadores ligados al subsector hullero, se habian mantenido en un delicado equilibrio durante el periodo autárquico que siguió al fin de la guerra española. Bajo el manto, supuestamente homogenizador, de la fuerte demanda de mano de obra de la minería del carbón se escondian múltiples realidades de distinto signo, pero con la suficiente entidad como para revelar que cualquier variación en la estructura económica del país podía afectar gravemente a cientos de miles de trabajadores y sus familias. En primer lugar, el sistema industrial hullero llegaba a finales de los años cincuenta acusando la ausencia de inversiones, un mal que si bien perseguia secularmente al subsector no dejaba de haberse acentuado durante los últimos veinte años; justo los que se tenían que haber empleado para poner al día las grandes unidades carboneras profundizadas en la segunda década del siglo. En segundo lugar, si bien era cierto que la demanda de brazos para las minas era aún importante, su intensidad había bajado respecto a la década anterior. Pero, además, el control político- policial, 
unos muy bajos salarios, y las duras condiciones de trabajo no eran el mejor acicate como para conseguir una respuesta masiva como la que se había obtenido en la posguerra. Y en tercer lugar, los desequilibrios urbanos y urbanísticos creados por la gran industria tampoco habían sido compensados por el sector público, que se había limitado a intentar paliar problemas como el de la vivienda obrera mediante los grandes polígonos residenciales que, sobre todo a fines de los cincuenta, empezaban a aparecer en la Cuenca Hullera Central asturiana.

Así que, los espacios de producción estaban obsoletos, los salarios eran tan bajos que no conseguian inducir la promoción inmobiliaria sobre unos ensanches trazados treinta años antes, y el medio ambiente urbano y rural tenían prácticamente los mismos servicios y comunicaciones que cincuenta años atrás, pese a haber soportado una sobrecarga demográfica que había hecho aparecer, tanto en la ciudad como en los pueblos auténticos focos de chabolismo. De este modo, todo parecía indicar que el sistema no tardaría mucho en desmoronarse, dentro de un contexto de mercados abiertos que exigian la tecnificación y la racionalización de la explotación del carbón: la solución adoptada tras la guerra de sustituir toda inversión en las minas por la aplicación masiva de factor trabajo, parecía volverse en contra del mismo sistema quince años después. Pero además otra razón cobraba fuerza dentro de las motivaciones sociales y territoriales que dieron lugar a los planes de mejora del hábitat minero: las reivindicaciones laborales. En efecto, tras la práctica aniquilación de las organizaciones obreras de los años cincuenta, en la década siguiente, y al calor de la reactivación económica y del fin al bloqueo salarial, el movimiento obrero empieza a dar señales de vida. Un tímido renacer continuamente golpeado por la bota represiva de la dictadura, que encuentra en los mineros asturianos el caldo de cultivo adecuado para su desarrollo, en tanto que secularmente los obreros de la minería siempre habian tenido que acudir a la huelga para conseguir mejoras salariales imprescindibles para la subsistencia. Así, la cuestión política, que en demasiadas ocasiones se ha puesto como premisa ineludible para la comprensión de la conflictividad laboral minera no deja de estar supeditada en la mayoría de momentos a algo mucho más pragmático como eran los motivos salariales, en especial en una década como la de los años sesenta donde la progresiva mayor oferta de productos y contacto con otras realidades chocaba con la congelación de unos salarios ya de por sí raquíticos. La década de los sesenta queda determinada en la minería asturiana de la hulla por las huelgas de 1962, continuamente revisadas y revisionadas desde ciertos planteamientos políticos hasta adquirir un corpus mitificado que coloca a los mineros solos al frente de la oposición al régimen gracias a su titánica convicción política. Sin embargo, no parece descabellado pensar que las condiciones salariales y las miseras condiciones de vida en buena parte de los territorios mineros y las ansias de obtener un cambio de las mismas, en este caso a través de la única herramienta de presión que había demostrado alguna validez como era la huelga, tenían un peso muy notable en el ideario de los huelguistas. En cualquier caso, las huelgas de comienzos de los años sesenta habian hecho saltar las alarmas dentro del régimen, de manera que una amplia masa de trabajadores, tradicionalmente reivindicativa, que habitaban la zona más poblada de la región, eran motivos más que suficientes para exigir que las medidas para la reestructuración de la minería de la hulla tuviesen en cuenta las personas y los territorios que la habian arrancado, bien fuera por cuestiones sociales, que las había incluso dentro del régimen, como de mera supervivencia del franquismo.

En este contexto, el 28 de junio de 1966 el Consejo de Ministros acordaba una serie de medidas "para la reestructuración del sector hulleron, entre las que figuraban unas disposiciones referentes a la Acción Concertada y otras para la mejora del Hábitat Minero. Este trabajo centra sus objetivos en el Plan Especial de Mejora del Hábitat Minero, y, por tanto, no es el lugar adecuado para analizar y valorar una cuestión tan decisiva y compleja como la Acción Concertada, cuyas disposiciones comienzan con el Plan de Desarrollo de 1963 y continúan en las bases generales de la Acción Concertada para el sector hullero de 1965. Sin embargo, lo que sí parece procedente es centrar someramente el periodo político y económico que va desde la liquidación de la Autarquía a la España del Desarrollismo. Un periodo que propició medidas como el Plan de Estabilización (1959) y los de Desarrollo (1964-67, 1968-1971, 1972-1975), sin los que la Acción Concertada y el Hábitat Minero nunca hubieran visto la luz.

\section{DE LA AUTARQUÍA AL DESARROLLISMO}

La imposibilidad de crecimiento de los distintos sectores productivos del país en un contexto autárquico fundamentado en una gran parafernalia ideológica de Nacional sindicalismo, cruzadas y románticas retóricas, agotaba la economía española por momentos. En efecto, pese a la creación de algunas plantas industriales modernas, como eran la siderúrgica integral de ENSIDESA, la constructora naval Bazán, 0 las de automoción de Seat o Pegaso, y pese al cortinaje de las grandes obras hidráulicas, el aislamiento internacional de Es-

1 La Acción Concertada es un proceso con abundantes referencias bibliográficas. Remitimos para una visión sintética de la cuestión, problemática y resultados a Díaz- Monasterio, F. (dir.) (1972): Acción Concertada, Ministerio de Hacienda, Servicio de Publicaciones, Madrid; Federación Estatal de Minería de la Unión General de Trabajadores (1981): El fraude de Hunosa, Editorial Adra, Madrid; Garcia Piñeiro, R. (1990): Los mineros asturianos bajo el franquismo 1937-1962, Fundación $1^{\circ}$ de Mayo, Madrid. 
paña impedía el desenvolvimiento natural de la economía y de sus sectores productivos, que no podían acudir al exterior para incorporar tecnología, conocimientos y capital para modernizar las estructuras industriales. Un parque industrial nítidamente subdesarrollado, donde la descapitalización secular se había acentuado tras la guerra española y europea parcheándose mediante la aplicación masiva de factor trabajo merced al bajo nivel de vida y las perentorias necesidades de la sociedad española.

Con este panorama, las medidas no podian hacerse esperar demasiado. Unas medidas que pasaban en primer lugar por el acercamiento a Estados Unidos como la incontestable gran potencia del mundo capitalista tras la II Guerra Mundial, lo que abrió a España las puertas de los organismos internacionales, diversas fuentes de financiación de vital importancia para la economía española de los cincuenta y preparó la progresiva apertura de mercados de la década siguiente ${ }^{2}$. Para todo ello, el inmovilista entramado político ideológico que inspiraba y controlaba la economía del franquismo ya no era válido, de manera que nuevas personas con mayor formación y un perfil más técnico, en muchas ocasiones insertos dentro de grupos no exentos de fuerte vinculación religiosa, fueron incorporándose a los distintos escalafones del gobierno. Así, el final de los años cincuenta y especialmente la década siguiente, coincidirán con un cambio más o menos radical en el núcleo del mensaje doctrinal del franquismo que, en definitiva, no dejaba de propiciar la perpetuación del régimen: del ideologizado bastión católico hispánico-imperial al sobresaliente y pragmático campeón del desarrollismo ${ }^{3}$.

Este cambio de política económica que se coordinaba, por tanto, con el de mensaje ideológico, venía gestándose durante toda la década de los cincuenta a través de las relaciones con Estados Unidos, principalmente, pero no acabaría tomando forma definitiva hasta el Decreto-Ley 10/1959 de 21 de julio de Ordenación Económica que inau- guraba, otra vez, una nueva España, la del Plan de Estabilización ${ }^{4}$. Un Plan de Estabilización que acapararía plenamente la nueva estrategia económica y política del franquismo pero cuyos planteamientos técnicos y normativos estuvieron directamente influenciados por los informes de organismos internacionales, como el Fondo Monetario Internacional (FMI) y la Organización Europea de Cooperación Económica (OECE), en el que España aspiraba a integrarse como una nación más, sin que su peculiar situación política pudiera siquiera cuestionarse ${ }^{5}$.

El Plan de Estabilización consiguió los objetivos de su enunciado: estabilizar la situación económica del país tras un largo periodo en el que la productividad, la compensación entre los factores de producción y el aumento o descenso de salarios, parecian seguir más unas líneas ideológicas que las normas elementales de la ciencia económica. No obstante, la estabilización y la consiguiente apertura progresiva de mercados ralentizó los sectores tradicionales poniendo al descubierto la situación de atraso de una industria nacional lastrada por décadas de ausencia de inversiones. Así, durante los años sesenta se acentuaron situaciones seculares de atraso del agro español y se cernieron sobre las regiones de antigua industrialización los fantasmas de la recesión, de la congelación de salarios y del paro. Son éstas, entre otras, algunas de las razones que llevaron a que se iniciara en España la planificación regional de la mano de los planes de desarrollo, cuyas bases se sentaban con la Ley 194/1963, de 28 de diciembre, por la que se aprobaba el Plan de Desarrollo Económico y Social para el periodo 1964-1967.

El Plan de Desarrollo contemplaba, al menos, dos cosas importantes para Asturias: los Polos y Polígonos Industriales, y el Régimen de Acción Concertada. En cualquier caso, las normas relativas a la ejecución del Plan de Desarrollo contenidas en el documento de aprobación y alcance (BOE del 30 de diciembre de 1963) estaban relacionadas unas con otras, en un intento de que la planificación consiguiera contener 0 ,

2 Sobre la modernización tecnológica experimentada en la industria española y asturiana como consecuencia de los acuerdos con Estados Unidos desde fines de la década de los cuarenta del siglo XX, véase SUÁREZ ANTUÑA, FAustino (2007). "Paisaje y producción: Los créditos y ayudas estadounidenses en la industria pesada asturiana a mediados del siglo XX". En I Congreso de Estudios Asturianos, Real Instituto de Estudios Asturianos, T. VII, Oviedo.

3 Fusı AIzPURÚA, J.P. (1985): "De la dictadura a la democracia. Introducción y La Década desarrollista», en Prado, J.M. (dir.): Historia de España, Plaza y Janés Editores, Barcelona, t. VII, pág. 105.

4 Sobre el Plan de Estabilización la bibliografia es muy extensa. Para aclarar de manera sencilla algunas cuestiones básicas, véase MARTín AcEÑA, P.; Comín, F. (1991): INI. 50 años de industrialización en España, Espasa Calpe, Madrid, pág. 118; y Sanz MenÉndeZ, L. (1997): Estado, Ciencia y tecnología en España: 1939-1997, Alianza Universidad, Madrid.

5 En junio de 1959 el Gobierno Español remitió a ambas instituciones un memorando que describia las medidas que la Administración española se comprometía a adoptar respecto al sector público, la política monetaria y el sector exterior (FEderACIÓN ESTATAL DE MINERíA, Op. Cit. págs 31). Tras la aprobación por estas instituciones de la ayuda financiera para la ejecución del Plan, a lo cual coadyuvó Estados Unidos, se aprobó el Decreto- Ley 10/1959 (SANZ MENÉNDEZ, 1997:129). En relación con los informes de estos organismos internacionales, Juan Pablo Fusı (1984: 107) apunta cómo fueron seguidos al pie de la letra - "como tablas de ley»- de los ministros desarrollistas. Una cuestión que, en el ámbito asturiano seguia plenamente vigente incluso en fechas tan tardías como 1964. En este año, el informe de la Sociedad Económica para la Conversión y Desarrollo Industrial (SODIC), como parte de la asistencia técnica de la OCDE (denominación que adquiriria la OECE desde 1960), acerca de la reorientación del sector secundario en Asturias sirvió, en buena medida, como directriz fundamental para la reordenación de la industria transformadora y su basculamiento hacia el área central (Sodic (1964): Las posibilidades de expansión de las industrias de transformación en la provincia de Oviedo, Diputación de Oviedo; y Carta remitida el 30/09/1966 por Mateu de Ros, Gobernador Civil de la Provincia de Oviedo, al Ministro Comisario del Plan de Desarrollo Económico y Social. Archivo Histórico de Asturias, Fondo Gobierno Civil, Caja 24678). 
FIG. 1: Problemas residenciales en las cuencas mineras asturianas a mediados de la década de los sesenta del siglo XX. Esta singular e inédita colección de imágenes ilustraba, tanto Plan Extraordinario de Obras y Servicios en las Cuencas Mineras como otros documentos y memorias elaborados por el Gobierno Civil y la Comisión Provincial de Servicios Técnicos en relación con los planes ordinarios y extraordinarios para Asturias. Y no era para menos. En ellas se ven con claridad algunos problemas que el Plan intentaba atajar: la degradación de los poblados más cercanos a las instalaciones mineras, el colapso de las estructuras poblacionales y residenciales heredadas por el aumento de población inmigrada, la conversión de instalaciones auxiliares del agro asturiano, como las cuadras, tenadas u hórreos, en infravivienda, y la perpetuación de focos de insalubridad, carentes de todos los servicios básicos, en torno a las vegas de las Cuencas. Las fotografías corresponden a: A) La Vega, El Entrego (SMRA); B) Camino a Pumarín de Abajo, Santa Ana, El Entrego (SMRA); C) Sueros (Mieres); D) Turón (Mieres). Comisión Provincial de Servicios Técnicos de Asturias (1966): Plan Extraordinario de Obras y Servicios en las Cuencas Mineras, Diputación Provincial de Asturias, Oviedo. Archivo Histórico de Asturias, Fondo Gobierno Civil, Caja. 24.663.

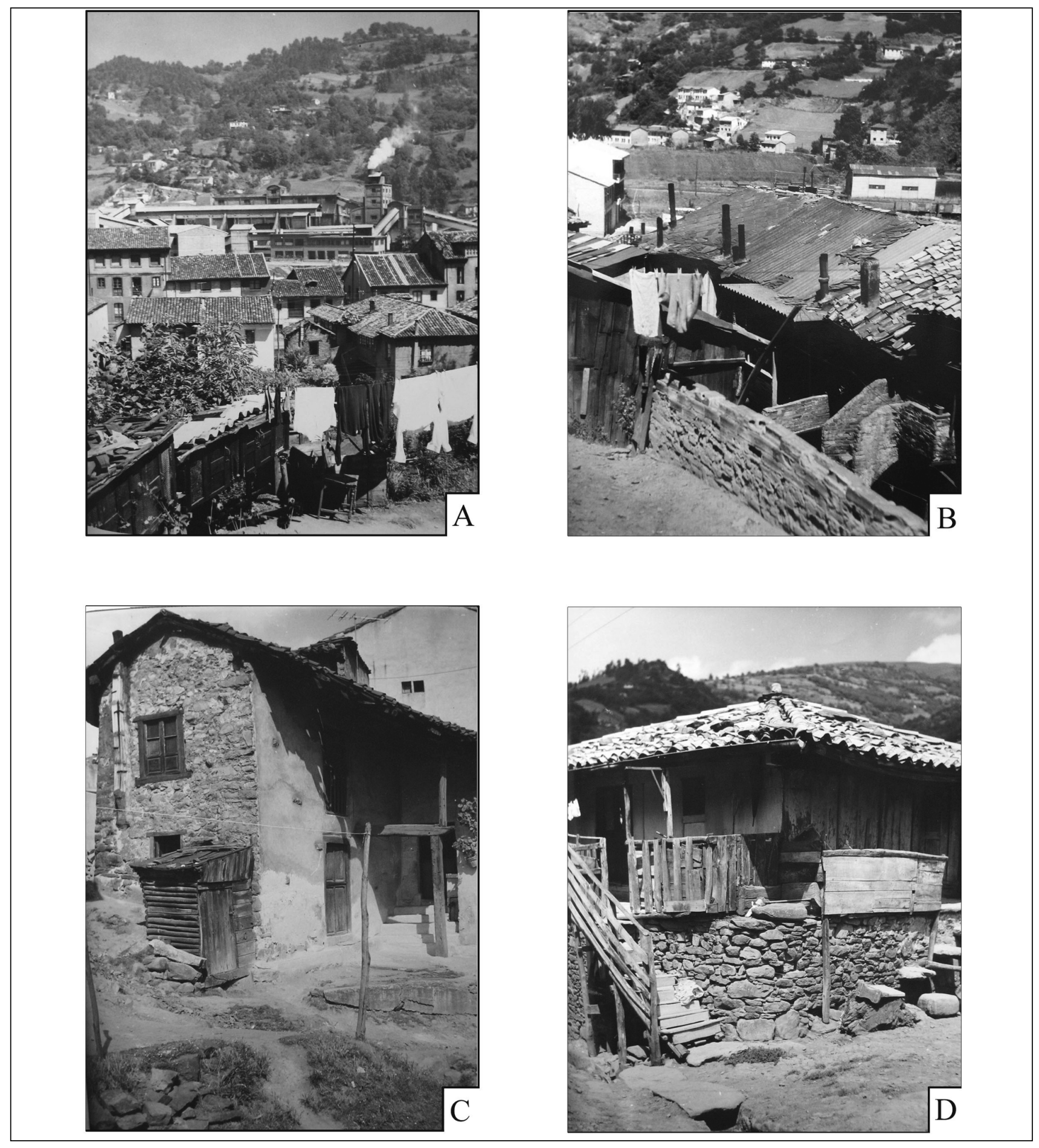


al menos, mitigar, los efectos que algunas medidas podian tener sobre la economía de los territorios afectados. En cuanto a los Polos y Polígonos, volveremos más adelante sobre ellos, en esencia contemplaban una serie de medidas fiscales y de suelo industrial encaminadas a la creación y potenciación de un sector secundario de mediano tamaño que sirviera como alternativa a los ajustes que se planteaban para las grandes empresas regionales del carbón y el acero, además de una ordenación de usos y funciones en el área central asturiana ${ }^{6}$. El régimen de Acción Concertada que se planteaba en el artículo $5^{\circ}$ del Plan de Desarrollo, no tendría materialización en la minería de la hulla hasta la Orden de Presidencia del Gobierno de 30 de marzo de 1965, en la que se fijaban las bases generales de la Acción Concertada para el subsector ${ }^{7}$. En ésta se recogían los objetivos del programa: "revalorización de la mineria», "reestructuración del sectorn, aumento de la productividad, instalaciones eficientes, racionalización de las explotaciones, concentración de unidades... En definitiva, lo que se pretendía era dar una ayuda a la minería para que mejorara sus estructuras productivas y que, de este modo, que pudiera tener un lugar en un contexto de mercado abierto.

\section{EL FIN DE LA AUTARQUÍA EN LAS COMARCAS MINERAS. LOS PELIGROS DE LA REESTRUCTURACIÓN}

Como es sabido, los efectos del régimen de Acción Concertada para la minería de la hulla fueron muy limitados, ante lo cual el Estado nacionaliza buena parte del subsector a través de Hulleras del Norte S.A (HunOSA), creada en 1967 para evitar el derrumbe económico y social de la región ${ }^{8}$. No obstante, bien fuera en manos privadas o públicas, lo que quedaba claro era que las cosas debian e iban a cambiar irremediablemente para la industria hullera ${ }^{9}$.

Con estas bases, no era descabellado valorar que unas comarcas superespecializadas en la producción de carbón acusarian de manera directa la reestructuración del sector, acentuando los desequilibrios territoriales que décadas de ausencia de inversiones habian ido definiendo en materia de comunicaciones, vivienda y calidad de vida.

En cuanto a las comunicaciones, el sistema económico basado en la minería del carbón y de la siderurgia estaba totalmente vinculado a la red ferroviaria (Ferrocarril de Langreo y Ferrocarril del Norte, integrado luego en RENFE), mientras que las carreteras de cohesión interna y externa de la Cuenca Hullera Central prácticamente no habian variado desde su traza decimonónica y las mejoras del periodo primorriverista; y eso teniendo en cuenta que un indicador del desarrollo del pais en los años sesenta era el aumento de la motorización. Respecto a la vivienda, los avances que la promoción oficial habian posibilitado sobre la población obrera de las cuencas desde fines de la década de los cincuenta principalmente, quedarian en entredicho por la reducción de las actividades de base, lo que no haría sino esperar un aumento de la infravivienda que no se había conseguido eliminar desde el aumento demográfico de estos territorios con la inmigración posterior a la guerra civil ${ }^{10}$. Paralelamente a ello,

6 Véase, Fernández García, F. (1984): "El polo de desarrollo de Oviedo», Ería, 7, Oviedo, págs. 135- 147.

7 No fue la minería de la hulla, ni incluso la del carbón, la única en recibir la ayuda del Estado mediante el régimen de Acción Concertada. Hubo conciertos similares con la industria de la harina, de la piel, de la pasta de papel, del ganado vacuno, de las conservas vegetales, con la siderurgia, y con la industria naval.

8 Si bien existen discrepancias entre los distintos autores, parece que buena parte de las empresas mineras se valieron, en parte por las presiones de los grandes bancos presentes en los Consejos de Administración de las compañías, de los créditos de la Acción Concertada para financiar las pérdidas de las compañias y no para reconvertir y reestructurar el sector (FEDERACIÓN ESTATAL, 1981, Op. Cit., págs. 43 y 81 y ss.). En ocasiones, incluso pudiera pensarse en programas concretos realizados para hinchar ficticiamente el valor de las instalaciones con vista al siguiente paso del Estado: la gestión directa, la nacionalización del sector. De hecho, Carlos Ferrer Salat (Presidente de la CEOE) reconocia en 1970 que propietarios de empresas mineras le habian dicho que de saber como el Estado iba a pagar las ruinosas minas, hubiesen comprado antes muchas otras (Actualidad Económica, 16/05/1970, citado por GARCí PIÑEIRO, R. (1990): Los mineros asturianos bajo el franquismo 1937-1962, Fundación $1^{\circ}$ de Mayo, Madrid). Por ello, uno de los problemas estructurales que arrastró HunosA desde su creación fue "la sobre valoración en la liquidación en el periodo de concentración o integración, que supuso una gran contrapartida económica para los propietarios de las empresas integradas y una espina difícil de arrancar para la joven empresa pública" "[eran] empresas [las integradas con evidentes sobre valoraciones] con una descapitalización clara y creciente, maquinaria escasa y obsoleta y técnicas de producción desfasadas» (COMIsIOnes ObreRAs. Sección SindiCAL de HUNOSA, (1985): Análisis de la situación de Hunosa y perspectivas de futuro, ejemplar mecanografiado, inédito, Oviedo, pág. 5). En esta línea, Juan de Lillo cifra en 7.147.417.590 pesetas el precio de la integración (De Lillo CuAdRAdo, J. (1978): Asturias: una crisis permanente, Ayalga Ediciones, Salinas, pág. 298). No obstante, esta cuestión no acaba de estar suficientemente aclarada. Sobre este particular. Schawrtz y González, afirman que a cambio de los activos entregados los empresarios sólo recibieron acciones luego amortizadas, si bien mantuvieron en sus manos los mejores activos (materiales, maquinaria, talleres). Véase SChAWRTZ, P.; GonzÁlEz, M.J. (1978): Una historia del Instituto Nacional de Industria (1941- 1976), Editorial Tecnos, Madrid, pág. 113.

9 La evolución y desarrollo de la industria hullera en Asturias ha generado una amplia bibliografía desde la década de los años setenta. En el último trabajo publicado hasta la fecha, SUÁREZ AnTuÑA, F. (2006): Carbón para España. La organización de los espacios hulleros asturianos, Ayuntamiento de Gijón, Consejería de Cultura, Comunicación Social y Turismo del Gobierno del Principado de Asturias, KRK Ediciones, Oviedo, se aportan las principales referencias acerca de la cuestión.

10 El Consejo Sindical de la Provincia de Oviedo alertaba en 1959 del grave problema de la vivienda en Asturias, que afectaba en especial a las áreas industriales como Mieres y Langreo. Pese a los esfuerzos de organismos oficiales como la Obra Sindical del Hogar (OSH), que había promovido más de tres mil viviendas en la región entre 1953 y 1956, los autores del informe cifraban el déficit en 54.092 viviendas que, teniendo en cuenta la expansión de los sectores industriales y el aumento demográfico de la provincia (las previsiones eran, aún, plenamente optimistas) no se paliaria con el ambicioso Plan de urgencia Social de Asturias, publicado en el BOE el 31/10/1958, y que planteaba la construcción de 50.000 viviendas en la región. Todavia en fechas tan cercanas a los desarrollistas años sesenta, el in- 
el tímido desarrollo inmobiliario de las promociones privadas, incentivado al calor de la ley del suelo y de la mejora de los salarios tras las reivindicaciones de comienzos de la década, corrían el peligro de ralentizarse ante una situación de incertidumbre laboral, lo que dejaría los principales ensanches de Langreo, San Martín o Mieres como solares perpetuos en medio de una ciudad nunca consolidada urbanisticamente. Y, finalmente, la calidad de vida era un concepto simplemente desconocido. En efecto, con una infancia que creía que el color y densidad natural de los ríos era el negro cargado de los schlams del lavado del carbón, con unas escombreras que habían degradado y cambiado la topografía de las vegas y las faldas de las montañas, con el smog permanente de las chimeneas de la siderurgia, la producción termoeléctrica y las cocinas de carbón de las casas, pensar en alcantarillados y traídas de agua modernas, telefonía, así como equipamientos deportivos, culturales y educacionales distintos a los heredados de la República, treinta años antes, era sencillamente un imposible ${ }^{11}$.

\section{LAS MEDIDAS COMPLEMENTARIAS DE MEJORA DEL HÁBITAT MINERO}

Teniendo en cuenta esta situación, las medidas acordadas por el Consejo de Ministros en 1966 para la reestructuración del sector hullero contemplaban, además de una revisión de las ayudas del régimen de Acción Concertada, líneas de actuación para la mejora del hábitat minero ${ }^{12}$. En relación con esto, el Consejo de Ministros, de acuerdo con los Ministerios de Obras Públicas y de Hacienda, acordaba que en las provincias hulleras, las Comisiones Provinciales de Servicios Técnicos dependientes del Gobierno Civil estudiasen y formulasen en los tres meses siguiente programas de las mejoras a introducir en los caminos, abastecimientos de aguas, saneamientos, teléfonos, electrificaciones, fuentes, mercados "y otros centros y servicios públicos que permitan mejorar el hábitat minero»13.

\subsection{La confección y aprobación del Plan}

Con el plazo fijado, la redacción del programa o Plan que proponer era una tarea ingente, teniendo en cuenta además que tras décadas de práctica sequía inversora del Estado, los municipios estarian ávidos de introducir toda clase de reclamaciones acerca de infraestructuras básicas. Para regular el acopio de información y propuestas, se estableció un método de trabajo consistente en tres partes: en primer lugar definir el ámbito de actuación; en segundo lugar, recabar de diferentes organismos oficiales, como el Servicio de Cooperación de la Diputación Provincial o la Cámara Minera, toda la información acerca de infraestructuras de ese ámbito de estudio; y en tercer lugar, solicitar de los distintos ayuntamientos afectados la corroboración de los datos anteriores y de sus propuestas para la mejora del hábitat minero.

El primer resultado de todo ello fue la delimitación del ámbito de actuación, que quedó fijado para los municipios de: Aller, Bimenes, Caso, Langreo, Laviana, Lena, Mieres, Morcín, Quirós, Riosa, San Martín del Rey Aurelio, Siero, Sobrescobio, y Teverga ${ }^{14}$.

Se trataba ésta de una zona que aglutinaba en torno al $30 \%$ de la población asturiana, y que coincidía con la parte meridional del llamado "ocho asturiano", la estructura territorial y socioeconómica que se había ido configurando desde comienzos del siglo $\mathrm{xx}$ y que tenía como ejes superiores Avilés y Gijón, en la base las cuencas del Caudal y del Nalón, y en el medio Oviedo como vértice político, económico y ad-

forme recogía realidades de creación y desarrollo de la infravivienda: «se da el caso que un obrero [inmigrado] en cuanto se encuentra colocado, arregla de cualquier forma un hórreo, un pajar o construye una chabola y llama inmediatamente a su familia, lo que origina un agravamiento del problema pues no es preciso señalar que la vida de estas familias se desarrolla de una manera infrahumanan (CoNSEJo Sindical de LA Provincia de OVIEDo (1959): Estructuras y posibilidades de desarrollo económico de Asturias, Consejo Económico Sindical Nacional, págs. 441 y ss.)

11 El propio Plan de Mejora del Hábitat Minero así lo reconocía, cuando describía en la memoria cómo en los poblados mineros, y en buena parte de las Comarcas Mineras en realidad, y pese a la densidad de población que soportaban no existian caminos ni carreteras, abastecimiento de aguas, saneamiento, electrificación... (Comisión Provincial de SERvicios Técnicos de Asturias (1966): Plan Extraordinario de Obras y Servicios en las Cuencas Mineras, Diputación Provincial de Asturias, Oviedo. Archivo Histórico de Asturias, Fondo Gobierno Civil, Caja. 24.663).

12 En esa reunión del Consejo de Ministros, se recogía algo tan fundamental para los trabajadores del sector como la necesidad de impulsar el Régimen Especial Minero de la Seguridad Social. (Carta remitida el 09/02/1967 por Mateu de Ros, Gobernador Civil de la Provincia de Oviedo, a varios Ministros y altos cargos políticos y administrativos del Estado. Archivo Histórico de Asturias, Fondo Gobierno Civil, Caja. 24.679). El Régimen Especial de la minería del carbón incluye aspectos decisivos y pioneros en la Seguridad Social española, como el planteamiento global acerca de las enfermedades profesionales (en especial de la silicosis), los salarios normalizados o los coeficientes reductores. El Régimen Especial de la minería del carbón debería esperar al Decreto 384/1969 de 17 de marzo, posteriormente modificado con el Decreto 298/1973 de 8 de febrero y Orden Ministerial de 3 de abril de 1973, para adecuarlo a la Ley 24/1972 de 21 de junio de financiación y perfeccionamiento del Régimen General de la Seguridad Social.

13 Comisión Provincial de Servicios Técnicos de Asturias (1966): Plan Extraordinario de Obras y Servicios en las Cuencas Mineras, Diputación Provincial de Asturias, Oviedo. Archivo Histórico de Asturias, Fondo Gobierno Civil, Caja. 24663.

14 Un ámbito que coincide plenamente con el fijado en el Plan Complementario de Reactivación de las Comarcas Mineras, los conocidos como "Fondos Mineros», de fines de los años noventa del siglo XX. En éste, se añadieron Oviedo y Gijón, en principio exclusivamente en las zonas mineras de Olloniego y La Camocha, y se matizó, en la misma línea, la participación de Siero a Carbayín y Lieres. Además, se incluyeron los concejos de Cangas del Nancea, Degaña e lbias que no figuraban en el Plan de los años sesenta por estar dirigido exclusivamente a la hulla; estas áreas tendrian que esperar al Plan de reestructuración del sector de la antracita de 1971 (BOE 18/03/1971) donde se recogía igualmente la mejora de su hábitat. 
Fig. 2: Concejos afectados por el Plan de Mejora del Hábitat Minero, en el área central de Asturias: 1) Siero; 2) Bimenes; 3) Langreo; 4) San Martín; 5) Morcín; 6) Laviana; 7) Riosa; 8) Mieres; 9) Sobrescobio; 10) Teverga; 11) Quirós; 12) Lena; 13) Aller; 14) Caso. En la imagen se representan los ríos que drenan la Cuenca Hullera Central: el Nalón, a través de los concejos de Caso, Sobrescobio, Laviana, SMRA, y Langreo; y el Caudal, que concentra la red hidrográfica del resto de los concejos del área antes de unirse al Nalón. Éste, cercano ya a su desembocadura recibe igualmente las aguas del Narcea, que organiza la red hidrográfica de la principal zona antracitera de Asturias.

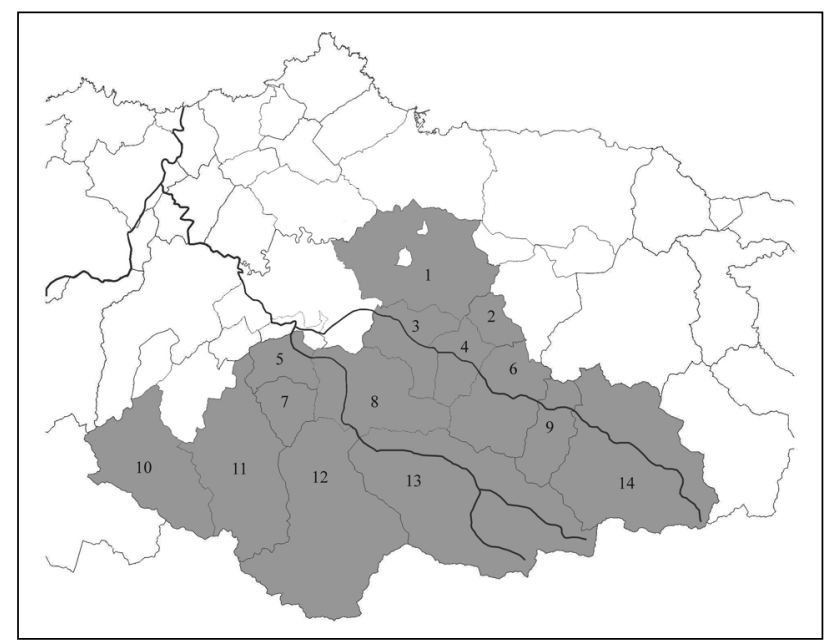

ministrativo de todo el sistema territorial. Por ello, la delimitación del ámbito del Plan a esos catorce concejos era una de las primeras constataciones de que el "ocho asturiano" tenía unas bases poco firmes, especializadas en unas industrias pesadas y con unas estructuras productivas anticuadas, como eran la siderurgia y la minería de la hulla, incapaces de competir en los nuevos tiempos que se abrian. Dos actividades abrazadas históricamente en un mismo territorio, pero que, ante el envite de la modernización económica emprendida por España, tendrian una evolución espacial distinta: la si- derurgia, liberada por la apertura de mercados del factor de localización que otorgaba el carbón autóctono, gozaria de mayor versatilidad en una localización costera, como la Unión de Siderúrgicas Asturianas (UNINSA); y el carbón, enraizado irremediablemente al territorio de referencia, llamado a modernizarse, a reducir y concentrar su estructura empresarial y productiva, y a dejar de ser el motor de Asturias ${ }^{15}$.

La selección de los ayuntamientos del Plan no fue decidida en exclusiva por el Gobierno Civil con el concurso de la Comisión provincial y la Diputación, sino que en ella habian colaborado, por ejemplo, la Cámara Minera de Oviedo, presidida entonces por Secundino Felgueroso, mediante un informe que valoraba en que zonas debía intensificarse el programa de mejora del hábitat en relación con las modificaciones que la población laboral podría experimentar en el marco de la reestructuración del sector hullero ${ }^{16}$. En cualquier caso, no fue esta una selección que fuera aceptada sin más por el resto de territorios mineros y limitrofes, que solicitaron infructuosamente su inclusión en el programa. Así, Degaña explicaba en 1967 cómo el 80\% de su población era minera, desarrollando su profesión en las minas de hulla de Coto Cortés (Grupos Mangueiro y Jatera, con 440 obreros) y Dos Amigos (Jaterina, 70 obreros) ${ }^{17}$. En el caso de Proaza, el ayuntamiento basaba su solicitud en el hecho de que, pese a no tener minas en su territorio, una parte muy importante de su población trabajaba en las existentes en los concejos de Teverga (Hullasa) y Quirós, configurando una zona de reclutamiento muy degradada que en algunos casos no poseían ni electrificación, caminos o telefonía.

En cuanto a la recogida de la documentación, en un primer momento, el Gobierno Civil encargó a la Diputación Provincial que realizara los estudios correspondientes a caminos y abastecimientos de aguas, mientras que los ayuntamientos debian remitirles los correspondientes a saneamientos y alcantarillados, teléfonos y electrificaciones, parques, urbanizaciones, centros sanitarios y equipamientos... ${ }^{18}$ Los ayunta-

15 La progresiva pérdida relativa de peso de las industrias de las cuencas respecto al conjunto regional, hace que éstas empiecen a asistir desde los años sesenta del siglo pasado a un proceso que no se había dado desde fines de la década de los diez: la pérdida de población en beneficio de áreas más pujantes. En definitiva, la actividad y su importancia en el conjunto regional, por tanto, basculaba hacia el norte haciendo que el ocho asturiano perdiera su eje meridional en beneficio del centro de la región. Esto daria paso a una nueva organización territorial que no es sino el reflejo del nuevo contexto socioeconómico: el triángulo Avilés- Gijón- Oviedo. Para estas cuestiones véase, Maurí Álvarez, M. (1992): «El territorio asturiano en el marco socioeconómico», en Morales Matos, G. (dir): Geografía de Asturias, Editorial Prensa Asturiana. T.I, págs. 66-80; y MURCIA NAVARRO, E. (1987): "Los efectos de la industrialización en la estructura territorial asturiana», Mineros, sindicalismo y política, Fundación José Barreiro, Oviedo, págs. 399- 425.

16 Desconocemos si tal informe llegó a realizarse, pero en la carta que el Gobernador Civil de la Provincia, José Manuel Mateu de Ros, enviaba a la Cámara Minera, el Gobernador Civil daba algunas pautas respecto a tal valoración: "conviene tengáis en cuenta que el hecho de concentrar los puntos de extracción no significa en muchos casos el trasplante de la población laboral minera de sus actuales zonas de residencia a los nuevos puntos concentrados de trabajo ya que esto se puede suplir perfectamente con adecuados medios de transporte, máxime teniendo en cuenta que el programa de nuevas viviendas, dado su coste y largo trámite, no iba a resolver de momento la creación de nuevos núcleos residenciales.n (Carta remitida por Mateu de Ros, Gobernador Civil de la Provincia de Oviedo, a la Cámara Minera de Oviedo. Archivo Histórico de Asturias, Fondo Gobierno Civil, Caja. 24.678)

17 Degaña remitía esta solicitud fuera de tiempo, tras haberse enterado del Plan por la prensa (La Nueva España 05/03/1967). Pese a reconocerse la justicia que lo acompañaba, se decidió integrar al concejo en el posterior plan promovido para la antracita del área del Nancea (Archivo Histórico de Asturias, Fondo Gobierno Civil, Caja. 24.680).

18 Estas cartas, que respondian a un modelo único para todos los ayuntamientos, terminaban encareciendo a los alcaldes el cumplimiento de esas instrucciones ya que de ello «depende en gran parte la mejora material y espiritual de nuestras zonas mineras». Quedaba claro, así, la doble intención del Plan. Correspondencia entre Gobierno Civil y ayuntamientos en relación con el Plan de Mejora del Hábitat Minero, julio de 1966. Archivo Histórico de Asturias, Fondo Gobierno Civil, Caja. 24.680. 
mientos respondieron con prontitud al requerimiento, planteando una larga serie de necesidades, acompañadas en algunas ocasiones de presupuestos, que la Comisión tendría que valorar para su inclusión en el Plan. Pero además del listado, los ayuntamientos solían enviar en muchos casos una carta adjunta de presentación donde exponían de manera general la situación del municipio y la necesidad de acometer aquellas obras. Se tratan estas comunicaciones de unos documentos de notable interés para aprehender la realidad cotidiana de estos territorios, al margen de las grandilocuentes manifestaciones públicas del régimen y con no poca dosis crítica en muchas de ellas. Así, por ejemplo, el Ayuntamiento de San Martín del Rey Aurelio remitía proyectos de caminos que ya habían sido contemplados por la Diputación años antes y explicaba que se trataban de obras, muchas de ellas, de poca envergadura, pero que podian contener en alguna medida el éxodo masivo de población desde el área rural a la urbana, que no era capaz de absorber en condiciones mínimas aquellos contingentes. Continuaba el ayuntamiento planteando que una serie de servicios destinados a mejorar la calidad urbana, como pavimentaciones, parques, jardines y casas de juventud, no podian seguir siendo consideradas como lujos ${ }^{19}$.

\subsection{Documento y tramitación del Plan}

El documento realizado por la Comisión Provincial de Servicios Técnicos de Asturias llevaba el título de Plan Extraordinario de Obras y Servicios en las Cuencas Mineras, siguiendo la denominación habitual de los planes provinciales pero remarcando su carácter extraordinario y, por tanto, adicional a aquellos. De esta manera, el Plan recogía una muy larga serie de actuaciones distribuidas de la siguiente manera:

Cuadro I. Distribución de los Fondos del documento del Plan Extraordinario

Tipo de obra Importe (ptas.) Número de obras

Caminos vecinales $142.700 .000 \quad 30$ Abastecimientos de agua 173.450 .000 30 Saneamientos
Tipo de obra Importe (ptas.) Número de obras

Electrificaciones, teléfonos, pavimentaciones y centro
de juventud
254.921.271
Total
843.850 .271
606

En octubre de 1966, Mateu de Ros, Gobernador Civil de Asturias, remitía a Laureano López Rodó, Ministro Comisario del Plan de Desarrollo, el Plan Extraordinario de Obras y Servicios en las Cuencas Mineras, empezando así la tramitación del documento. En los dias siguientes, el Gobernador Civil escribiría a numerosos altos cargos de la Administración y del Movimiento Nacional (Ministros, Secretarios, Subsecretarios, Directores Generales...) solicitándoles apoyo para el proyecto asturiano a sabiendas que muchos de ellos participarían con sus informes en el proceso de aprobación, asignación económica y puesta en marcha del Plan ${ }^{20}$.

En marzo de 1967, el Presidente de la Comisión Interministerial de Planes Provinciales de la Secretaría Técnica de la Presidencia del Gobierno elevaba el Plan Extraordinario al Ministro Subsecretario de la Presidencia del Gobierno, con informe y propuesta, para su aprobación por el Consejo de Ministros. En el informe, se llegaba a una serie de conclusiones: en primer lugar se estimaba la urgencia y conveniencia del Plan e incluso se hacía eco de la utilidad de construcción de un nuevo y moderno vial que uniera Langreo y Oviedo, pese a que no se hubiera incluido en el Plan ${ }^{21}$; en segundo lugar, se señalaba la conveniencia de que el Plan se acomodara a las acciones que se emprendieran dentro del II Plan de Desarrollo; y en tercer lugar se cifraba el coste del Plan en 512 millones de pesetas que se distribuirian en tres anualidades entre 1967 y 1969 (125 millones en la primera y 193,5 en las otras dos) ${ }^{22}$. En el cuadro II puede observarse la diferencia entre lo presupuestado en el Plan elaborado por la Comisión de Servicios Técnicos de Asturias y lo finalmente aprobado:

CUADRO II. Fondos solicitados y concedidos.

$\begin{array}{lll}\text { Tipo de obra } & \begin{array}{l}\text { Presupuesto } \\ \text { Comisión Provincial }\end{array} & \begin{array}{l}\text { Presupuesto } \\ \text { Aprobado }\end{array} \\ \text { Caminos vecinales } & 142.700 .000 & 317.000 .000\end{array}$

19 Memoria remitida por el Ayuntamiento de San Martín del rey Aurelio al Gobierno Civil en julio de 1966 en relación con el Plan de Mejora del Hábitat Minero. Archivo Histórico de Asturias, Fondo Gobierno Civil, Caja. 24.680. Igualmente interesante resulta la lectura de la moción aprobada por el Ayuntamiento de Langreo en julio de 1966 e incluida en el memorando remitido al Gobierno Civil, respecto al futuro industrial de Langreo ante la crisis de los sectores tradicionales. Archivo Histórico de Asturias, Fondo Gobierno Civil, Caja. 24.681.

20 Este es el caso de Pedro Areitio y Rodrigo, Director General de Carreteras y Caminos Vecinales del Ministerio de Obras Públicas, que contestaba a Mateu alabando la confección del Plan pero también haciéndole partícipe del informe que había elevado al Ministerio. En este informe, Areitio objetaba que 384 kilómetros de los nuevos caminos planteados tenían carácter de vías provinciales y que, por tanto, debian estar desde su construcción a cargo de la Diputación Provincial y no de un plan extraordinario.

21 Se trata de la carretera entre Riaño (Langreo) y San Miguel de La Barreda (Siero), hoy denominada AS-17 (Avilés- Puerto de Tarna), que no se construiria hasta mediada la década siguiente y que durante más de treinta años fue prácticamente la única vía de conexión real entre la cuenca de Langreo y el centro de Asturias.

22 Informe que el Presidente de la Comisión Interministerial de Planes Provinciales de la Secretaría Técnica de la Presidencia del Gobierno eleva al Ministro Subsecretario de la Presidencia del Gobierno con relación al Plan Extraordinario de Obras y Servicios en las Cuencas Mineras, 2 de marzo de 1967. Archivo Histórico de Asturias, Fondo Gobierno Civil, Caja. 24.678. 
Tipo de obra

Abastecimientos de agua

Saneamientos

Electrificaciones (1),

teléfonos (2),

pavimentaciones y centros

de juventud (3)

\subsubsection{1}

Total

843.850 .271

272.779 .00
Presupuesto

Aprobado

Comisión Provincia

128.000 .000

$5.438 .820(1)$

$8.375 .000(2)$

$53.186 .180(3)$

512.000 .000

Una diferencia que sólo superaba a la valoración del Plan en la categoría de los caminos, pero que veía reducirse en más de un 70\% cada uno de los restantes apartados. De esta manera, el Plan Extraordinario de Obras y Servicios en las Cuencas Mineras de Asturias nacía con casi un 40\% menos de dinero que el estimado inicialmente, con la necesidad de que se integrara en las políticas de planificación económica del Plan de Desarrollo y que, además, contara con la financiación propia de la Diputación y de los Ayuntamientos. Eran todas estas unas cuestiones de suma importancia y complejidad, que no hacian sino dificultar, cuando no impedir, que el Plan se convirtiera en el revulsivo para la calidad de vida que se había buscado con su redacción. En efecto, una de las maneras de conectar el Plan Extraordinario de las Cuencas Mineras con el Plan de Desarrollo eran las Zonas de Localización Industrial y los Polígonos Industriales. Una cuestión que había sido solicitada reiteradamente por parte del Consejo Económico Sindical Provincial y avalada por los estudios del SODIC y de la Sociedad Asturiana de Estudios Económicos e Industriales (SADEI) y que no había tenido ningún fruto hasta el momento ${ }^{23}$. Respecto a la financiación del Plan por parte de la Diputación y los Ayuntamientos, esta fue una de las partes más complejas del Plan y, asi figura en la memoria de seguimiento del mismo en 1968, donde la Comisión Provincial de Servicios Técnicos exponía cómo las corporaciones locales no tenían medios para desarrollar prácticamente ninguna obra y, por ello, algunas de las proyectadas no podrian desarrollarse. Por ello, proponían que, o se aumentaba la consignación presupuestaria de los consistorios, o el Estado asumía el sobrecargo de las obras, liberando a los ayuntamientos de la carga solidaria de las obras del Plan, o bien se planteaba un aumento significativo de la dotación del II Plan de Desarrollo para que, en el transcurso de tres años, llevara "los modernos elementos de vida a todos los pueblos de Españan.

\subsection{Las obras del Plan Extraordinario}

El Plan Extraordinario de Obras y Servicios en las Cuencas Mineras de Asturias, pese a la merma de presupuesto, posibilitó que se efectuaran numerosas infraestructuras básicas.

En efecto, todos los municipios del ámbito de aplicación vieron como se acometieron traídas de aguas a numerosos pueblos, se trazaron y construyeron caminos y carreteras que conectaban y vertebraban el término municipal, se sanearon y dragaron algunos ríos, se construyeron colectores, se llevó a cabo una gran obra de electrificación rural y de pavimentaciones, olvidadas durante decenios, se instalaron numerosos teléfonos y se construyeron instalaciones juveniles y deportivas.

Respecto a la redacción de los proyectos, mientras que los de abastecimiento y saneamiento fueron encargados a la

FIG. 3. Distribución de las inversiones del Plan de Mejora del Hábitat Minero por concejos

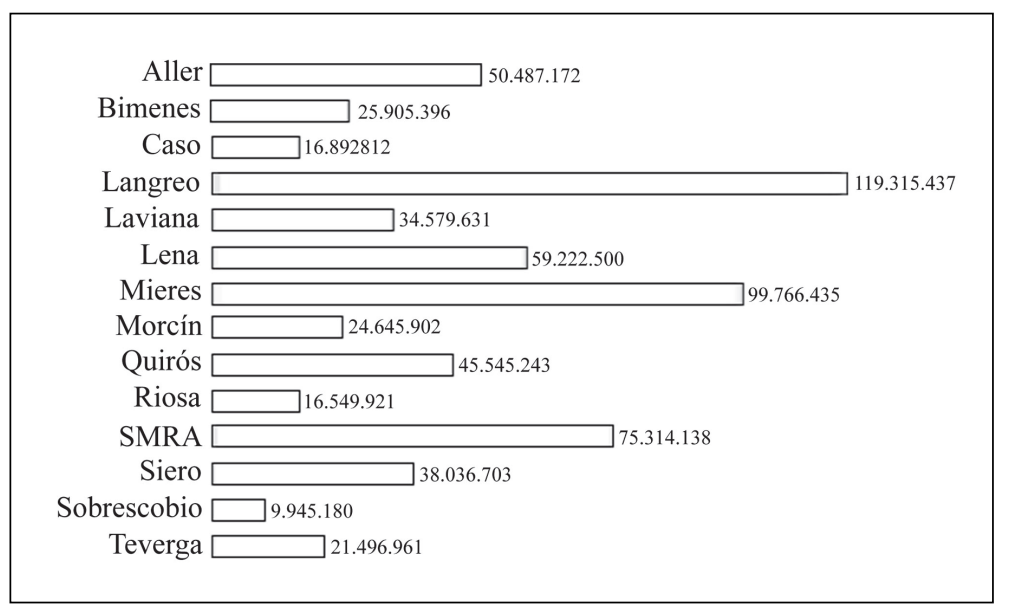

23 La cuestión de las Zonas de Localización Industrial Preferente, así como la falta de vivienda obrera de promoción pública, la falta del Régimen Especial Minero y el papel que debían tener las empresas públicas en la formación, entre otros temas que como estos dormían el sueño de los justos pese a su reconocimiento específico por parte del Consejo de Ministros, eran tratados de manera muy clara y crítica por Mateu de Ros, Gobernador Civil de Asturias, en una carta remitida al Vicepresidente del Gobierno, y varios ministros en febrero de 1967 (Archivo Histórico de Asturias, Fondo Gobierno Civil, Caja. 24.679). 
Confederación Hidrográfica, y los de electrificación, telefonía, caminos y carreteras los realizó la Diputación Provincial, los destinados a casas de juventud y polideportivos se llevaron directamente desde el Gobierno Civil. Estos proyectos deportivos tenían, si cabe, una singularidad aún mayor, ya que, en realidad, se trataba de la primera vez que existían este tipo de equipamientos en la Cuenca Hullera Central y porque, durante varias décadas, fueron las únicas instalaciones deportivas dignas con que contaron los municipios mineros. No obstante, no tuvieron un desarrollo sencillo, ya que la merma del presupuesto impedía la construcción de los necesarios, por lo que se incorporaron financieramente al Plan de Urgencia de Instalaciones Deportivas de la Provincia. Una cuestión que venía a incidir nuevamente en la pérdida progresiva de la adicionalidad con que había nacido el Plan de mejora del Hábitat Minero, pero que ante las barreras económicas y burocráticas de la Administración fue la moneda de cambio para realizar buena parte de las obras proyectadas. A este respecto, el Gobernador Civil de Asturias, Mateu de Ros, consiguió implicar al entonces Delegado Nacional de Educación Física, Juan Antonio Samaranch, para la realización del proyecto base y, luego, para una importante ayuda financiera ${ }^{24}$.

No obstante, la construcción de las instalaciones deportivas causó varios problemas con las corporaciones locales. En algunos casos, como en San Martín del Rey Aurelio, la pretensión inicial de construir dos instalaciones hubo de reducirse a una, la de El Entrego, que quedaba encuadrada en las de mayor envergadura, la de tipo $A$, mientras que la que daría servicio a la villa capitalina de Sotrondio y Blimea, sería financiada por el consistorio, rebajándose su categoría a la de tipo B. En otros casos, como en Siero y Langreo, los ayuntamientos no acababan de aportar terrenos libres de cargas y de superficie necesaria para el desarrollo de los proyectos, habitualmente por problemas en la adquisición y/o expropiación del suelo ${ }^{25}$.

\section{CONCLUSIONES}

En un tiempo en el que los planes complementarios para la reactivación de las Comarcas Mineras siguen copando, como desde hace casi una década, buena parte de la información y debate político en las Cuencas y en Asturias, puede ser interesante echar la vista atrás y ver qué parte de las novedades del presente se enraízan en el pasado; observar y tomar nota de cómo los programas que existieron con anterioridad se encontraron, o no, con problemas que hoy son de tanta actualidad, y en que medida se fueron sacando adelante los distintos planteamientos. A lo largo de este trabajo hemos pretendido ofrecer un primer acercamiento a una serie de medidas extremadamente novedosas en el contexto histórico en que se desarrollaron y que no habian sido tratadas en ningún modo hasta el momento. Fueron unas medidas que, en efecto, llegaban tarde, por lo que no pudieron atajar los ambiciosos fines con que habian nacido, y cuyo desarrollo se prolongaría en exceso, en muchos casos más de una década. Pero lo que no se puede negar es que sus efectos fueron inmediatos y positivos.

FIG. 4. Proyecto de polideportivo del Plan de mejora del Hábitat Minero firmado por Carlos Blanco Bescos.

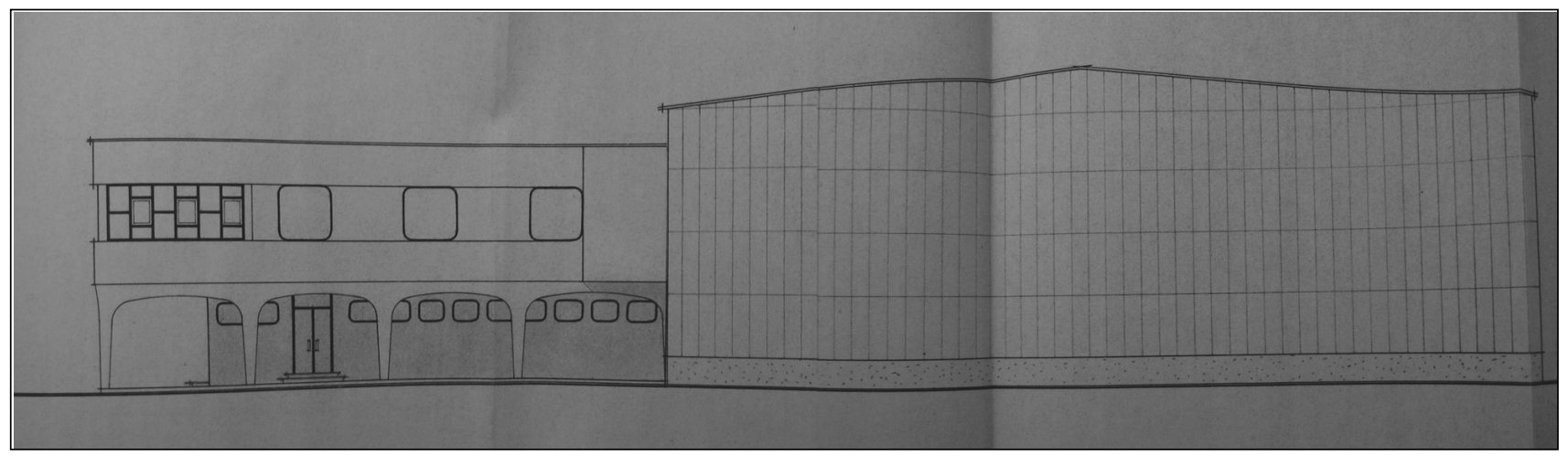

24 A este respecto, con los dos millones y medio para cada instalación que se preveía en el proyecto inicial, era, en palabras de Samaranch "casi imposible realizar una instalación de condiciones mínimas". El anteproyecto redactado por la Delegación Nacional de Deportes fijaba el presupuesto en 6.450 .000 pesetas, que sería rebajado a cuatro millones en el proyecto redactado por el arquitecto de la Diputación Provincial, Manuel Bobes. En cualquier caso, los proyectos deportivos finales están firmados por el arquitecto de la Junta Provincial de Educación Física y Deportes, Carlos Blanco Bescos (Archivo Histórico de Asturias, Fondo Gobierno Civil, Caja. 24.680).

25 En el caso de Siero, la construcción de la cancha polideportiva en Lieres topaba con el hecho de que parte de los terrenos estaban ocupados por las instalaciones de una alcoholera, que habia nacido sobre una antigua azucarera. Habia, por tanto, que adquirir los terrenos a Azucarera Española y derribar para aportar los terrenos convenientemente al Plan. (Archivo Municipal del Ayuntamiento de Siero. Expediente sobre la alcoholera de Lieres). En cuanto a Langreo, el problema que surgía era que la construcción del polideportivo se pretendia hacer en unos terrenos calificados en el planeamiento como parque, ante lo que los propietarios exigian un aumento en el precio de compra (Archivo Histórico de Asturias, Fondo Gobierno Civil, Caja. 24.680). 
El Plan de Mejora del Hábitat Minero o, utilizando la terminología del documento asturiano, el Plan Extraordinario de Obras y Servicios en las Cuencas Mineras, de 1966 supuso el primer gran proyecto para las cuencas mineras de la hulla asturiana. Un proyecto liderado por el entonces Gobernador Civil de la provincia y Jefe Provincial del Movimiento, José Manuel Mateu de Ros, que puso todo su empeño, capacidad y relaciones para que se llevaran a cabo las electrificaciones y telefonías olvidadas, los caminos, traídas, pavimentaciones o saneamientos pendientes, pero también polideportivos y casas de juventud prácticamente impensables para el momento. En efecto, el Plan sirvió para eso, pero también para mostrar que los problemas tenian una raíz más profunda, que se anclaba en el anquilosamiento de unas estructuras productivas y políticas incapaces de asistir a los cambios que se avecinaban y cuya única tabla de salvación, tanto para los obreros como para los empresarios y en buena medida para la región, era la nacionalización de la hulla y el acero. Casi cuarenta años después, cuando el modelo público de gestión industrial heredado empieza a tocar a su fin mediante la tremenda reducción de la actividad, se vuelve nuevamente a la necesidad de mejorar el hábitat minero. Se retoman entonces, de la mano de los fondos de compensación y reactivación de las Comarcas Mineras (programas de planteamientos muy similares a los de entonces) saneamientos, urbanizaciones, comunicaciones, proyectos culturales...Salta a la vista, ayer como hoy, el excesivo protagonismo de las vías de comunicación respecto al resto de proyectos, en parte porque políticamente siempre ha sido más sencillo y rentable idear una carretera que afrontar problemas de mayor calado ${ }^{26}$. De la misma manera, vuelven a la actualidad viejas polémicas, como la adicionalidad o complementariedad de los fondos asignados, la gestión de los fondos y de las obras...

Pero, además de todo lo anterior, vuelven a salir a la luz conceptos que deben ser revisados de una manera crítica. Así, en los documentos del Plan de Mejora del Hábitat Minero de los años sesenta, había continuas referencias al trabajo de los mineros y siderúrgicos por el bien y el desarro-
Ilo de la patria, la contribución de éstos al país cuando éste más lo necesitaba... en definitiva, a la deuda histórica que con ellos se tenía. Un concepto que aún hoy sigue funcionando política y popularmente pese a la injusticia de su planteamiento y su origen predemocrático, anclado en el más recalcitrante paternalismo estatal de posguerra. Porque no se puede entender por qué los mineros y siderúrgicos contribuyeron más al desarrollo del país que el resto de los trabajadores de España, con los que compartían no sólo la sujeción absoluta al patrón y el no ser dueños de los medios de producción, sino también su fin último: trabajar, vivir y alimentar a su prole. Un concepto que, por tanto, debe ser abandonado de una vez por todas para abrazar otro como el de la justicia territorial, el de la acción equilibradora del Estado, que impida que queden territorios abandonados a su suerte, desestructurados espacial y económicamente, sin necesidad de deudas históricas más allá de las de la memoria. Este, y no otros conceptos, son la clave para lo que se demanda $y$, además, sí que están en la base de nuestro Estado de Derecho.

Las obras desarrolladas con cargo al Plan Extraordinario de Obras y Servicios en las Cuencas Mineras de Asturias, pese a la merma de presupuesto $y$, en muchos casos, la tardanza de financiación y ejecución por la centralización del Estado y la lejanía de la toma de decisiones, supusieron un punto de inflexión en la mejora de las condiciones de las zonas hulleras de Asturias. Un punto de inflexión que ha caído en el olvido más absoluto, pero que ha tardado décadas en poder superarse a través de nuevas líneas de financiación, como las Zonas de Urgente Reindustrialización (ZUR) de promoción económica e industrial en los años ochenta, como el Plan Nacional de Interés Comunitario (PNIC) que posibilitó el saneamiento y construcción de colectores generales en la década de los noventa, y el Plan Complementario de Reactivación de las Comarcas Mineras, gracias al cual todavía se siguen ejecutando obras importantes para el desarrollo económico y mejora de la calidad de la Cuenca Hullera Central, pero también, no lo olvidemos, para la vertebración de una región que lucha por modernizarse.

26 En las Jornadas de Recuperación Económica de las Comarcas Mineras celebradas en 1987, se señalaba que era "urgente, primero, construir carreteras que unan rápidamente los valles con los puertos para integrarlos en la Asturias central; es urgente, además, limpiar la suciedad que otros dejaron, recuperando los espacios medioambientales y rehabilitando el patrimonio industrial abandonado [más adelante dirá que con un patrimonio industrial restaurado se hará de la historia economia]; y, por fin, debemos crear servicios culturales para el ocio y suelos industriales para el negocio: las ventajas de localización estarán entonces en las cuencas». OJEDA, G. (1987): «Las Comarcas Mineras en la economia asturiana", en Jornadas de Recuperación Económica de las Comarcas Mineras, SOMA-UGT, El Entrego, pág. 31. 